\title{
Berechnung von Nichtlinearitätsparametern von RF MOS Mischern mittels Volterra-Reihen
}

\author{
A. Susic, A. H. Darrat, and W. Mathis \\ Leibniz Universität Hannover, Institut für Theoretische Elektrotechnik, Appelstr. 9A, 30167 Hannover, Germany
}

\begin{abstract}
Zusammenfassung. Die Nichtlinearität einer Mischerschaltung bezüglich des Informationssignals führt zu unerwünschten Spektralanteilen am Ausgang des Mischers. Da nicht alle Spektralanteile kritisch sind, müssen bei einer Nichtlinearitätsanalyse nur bestimmte Spektralanteile ermittelt werden. In dieser Arbeit wird ein Verfahren zur Bestimmung der Spektralanteile der Zustandsgrößen im Mischer mit Hilfe der Volterra-Reihe gezeigt. Das Verfahren basiert auf die Methode der nichtlinearen Stromquellen. Es wird sowohl auf nichtschaltende als auch schaltende Mischer angewendet. Hierbei wird der erste als ein zeitinvariantes System mit zwei Eingängen und der zweite als ein periodisch zeitvariantes System mit einem Eingang modelliert. Das Verfahren wird in dem Computeralgebraprogramm MAPLE für den einfach balancierten MOS Mischer implementiert. Es ermöglicht die Herleitung von semi-symbolischen Ausdrücken für die Spektralanteile in Abhängigkeit von den Entwurfsparametern. Die numerischen Ergebnisse des Verfahrens werden gegenüber Simulationen mit SpectreRF verglichen.
\end{abstract}

\section{Einleitung}

Ein idealer Mischer kann im Allgemeinen als ein Multiplizierer gefolgt von einem Filter betrachtet werden. Hierbei wird das Informationssignal mit einem vom Lokaloszillator generierten Hilfssignal (das LO-Signal), um deren Frequenz ab- bzw. aufwärts gemischt werden soll, multipliziert. Bei multiplikativen Mischern werden das Informationssignal (das RF-Signal) und das LO-Signal an separaten Eingängen eingeführt. Abhängig von der Größe des LO-Signals kann zwischen schaltenden (commutating) und nicht-schaltenden Mischern unterschieden werden. Ein nicht-schaltender Mischer kann als ein zeitinvariantes schwach nichtlineares System mit zwei Eingängen beschrieben werden, während ein

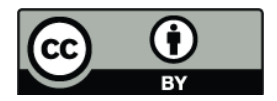

Correspondence to: W. Mathis (mathis@tet.uni-hannover.de) schaltender Mischer als ein periodisch zeitvariantes schwach nichtlineares System beschrieben wird.

Die Nichtlinearität des realen Mischers bezüglich des Informationssignals führt zu unerwünschten Spektralanteilen am Ausgang des Mischers (siehe Abb. 1). Da der Mischerausgang gefiltert wird, sind nur die Spektralanteile am Ausgang kritisch, die innerhalb des Durchlassbereiches des Filters liegen. Demzufolge müssen bei der Nichtlinearitätsanalyse nur die Spektralanteile bestimmt werden, die innerhalb des Durchlassbereiches des Filters liegen. Um diese unerwünschten Spektralanteile zu bestimmen ist eine Nichtlinearitätsanalyse der entsprechenden Mischerschaltung nötig.

Die Volterra-Reihe (Schetzen, 1980) hat sich als ein effizienter Ansatz zur Analyse dynamischer schwach nichtlinearer Systeme erwiesen. Die beiden Methoden zur Analyse nichtinearer Systeme, die auf der Volterra-Reihe basieren, sind die so genannte „Probing Method“ (Bedrosian und Rice, 1971) und die Methode der nichtlinearen Stromquellen (Bussgang et al., 1974; Schetzen, 1985). Wobei bei der ersten Methode die nichtlinearen Übertragungsfunktionen des Systems (Volterra Kernels) bestimmt werden, ermöglicht die zweite Methode die Antworten des Systems auf bestimmte Anregungen zu ermitteln, ohne die nichtlinearen Übertragungsfunktionen bestimmen zu müssen. Die Methode der nichtlinearen Stromquellen ist insbesondere bei Systemen mit mehreren Eingängen vom Vorteil, wo die Volterra Kernels zu Tensoren werden. Eine Anwendung dieser Methode auf schwach nichtlineare zeitinvariante Systeme mit mehreren Eingängen ist in Wambcaq und Sansen (1998) gezeigt.

Im Jahre 1978 präsentierte Swerdlow (Swerdlow, 1978) basierend auf der zeitvarianten Volterra Reihe (Flake, 1963) ein Verfahren zur Analyse von Intermodulationsverzerrungen bei additiven Mischern mit einem Pumpsignal. Hierbei wurde das System als periodisch zeitvariant modelliert und die resultierenden zeitvarianten Volterra-Kernels bestimmt. 1987 kombinierte Maas (Maas, 1987) die Methode der nichtlinearen Stromquellen mit der Methode der Konversionmatrizen (Oberg, 1973) zur Analyse von Intermodulationsverzerrungen bei Diodenmischern. Das Verfahren erlaubt die

Published by Copernicus Publications on behalf of the URSI Landesausschuss in der Bundesrepublik Deutschland e.V. 


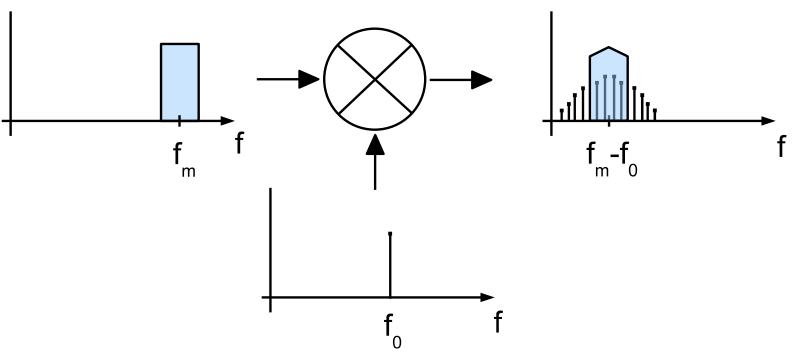

Abb. 1. Unerwünschte Spektralanteile am Ausgang auf Grund der Nichtlinearität des Mischers.

Bestimmung der resultierenden Spektralanteile für jede Knotenspannung und Zweigstrom im Netzwerk.

In dieser Arbeit wird ein Verfahren zur Nichtlinearitätsanalyse von einfach balancierten MOS Mischern präsentiert. Es wird dabei sowohl der nicht-schaltende Fall als auch der schaltende Fall betrachtet. Ziel dieser Arbeit ist es, semi-symbolische Ausdrücke der Spektralanteile und Nichtlinearitätsparameter in Ahbängigkeit von den Entwurfs- und Prozessparametern zu bekommen. Da das Verfahren eine Berechnung der Spektralanteile an jeder Knotenspannung und jedem Zweigstrom ermöglicht, gibt es dem Designer einen Einblick über die Entstehung der unerwünschten Spektralanteile im Netzwerk. Das Verfahren wurde in dem Computeralgebra-Programm MAPLE implementiert.

\section{Die Methode der nichtlinearen Stromquellen}

Die Volterra-Reihe beschreibt die Beziehung zwischen dem Ausgang und Eingang eines zeitinvarianten nichtlinearen dynamischen Systems in der folgenden Form (Schetzen, 1980):

$y(t)=H_{1}[x(t)]+H_{2}[x(t)]+\ldots+H_{n}[x(t)]+\ldots$

mit

$\mathrm{H}_{\mathrm{n}}[x(t)]=\int_{-\infty}^{+\infty} \ldots \int_{-\infty}^{+\infty} h_{n}\left(\tau_{1}, \ldots, \tau_{n}\right) x\left(t-\tau_{1}\right) \ldots x\left(t-\tau_{n}\right) d \tau_{1} \ldots d \tau_{n}$

wobei $H_{n}[x(t)]$ ein mehrdimensionales Faltungsintegral repräsentiert und Volterraoperator $n$-ter Ordnung genannt wird. Die Funktion $h_{n}\left(\tau_{1}, \ldots, \tau_{n}\right)$ in Gln. (2) ist als mehrdimensionale Impulsantwort definiert und wird Volterrakern $n$-ter Ordnung genannt. Somit kann der Ausgang eines nichtlinearen Systems durch eine Summe von mehrdimensionalen Faltungsintegralen des Eingangssignals dargestellt werden (siehe Abb. 2).

Ausgehend von Gln. (1) kann die Antwort des nichtlinearen Systems mit der Ausgangsgröße $\tilde{y}(t)$ als Summe von

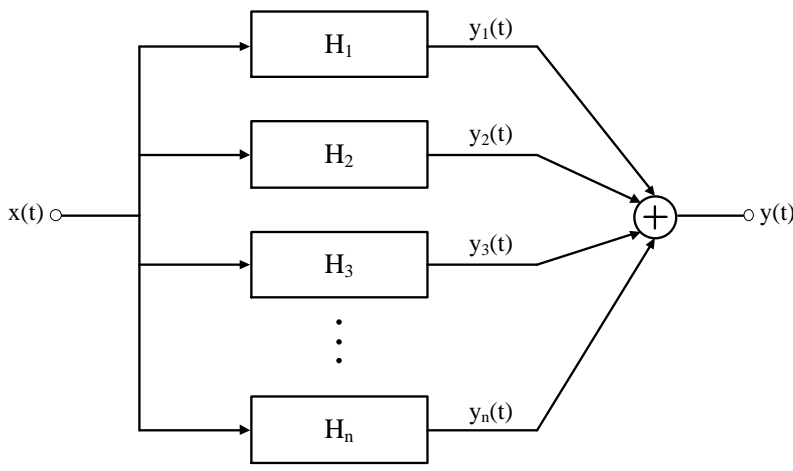

Abb. 2. Darstellung eines nichtlinearen Systems mittels der Volterra-Reihe.

mehreren Antworten höherer Ordnungen $\tilde{y}_{n}(t)$ angenähert werden.

$\tilde{y}(t) \approx \sum_{n=1}^{N} \tilde{y}_{n}(t)$

Lässt sich die Ausgangsgröße $\tilde{y}(t)$ des nichtlinearen Systems in Abgängigkeit der Eingangsgröße $\tilde{x}(t)$ um den Arbeitspunkt $\left(x_{0}, y_{0}\right)$ in einer Potenzreihe der Form

$\tilde{y}(t)=\sum_{n=1}^{N} a_{n} \tilde{x}^{n}(t)$

entwickeln, wobei $a_{n}$ der Taylor-Koeffizient $n$-ter Ordnung ist, und formuliert man die Ein- und Ausgangsgrößen in einer Potenzreihe eines „Sortierfaktors“ $c$ um

$\tilde{y}(t)=\sum_{n=1}^{N} c^{n} \tilde{y}_{n}(t) \quad$ und $\quad \tilde{x}(t)=\sum_{n=1}^{N} c^{n} \tilde{x}_{n}(t)$,

so kann nach Vergleich der Koeffizienten von $c^{n}$ jede Antwort $n$-ter Ordnung $\tilde{y}_{n}(t)$ in Abhängigkeit der Komponenten niedrigerer Ordnungen der Eingangsgröße $\tilde{x}$ ausgedrückt werden. Für die Antworten der ersten drei Ordnungen gilt:

$$
\begin{aligned}
& \tilde{y}_{1}(t)=a_{1} \tilde{x}_{1}(t) \\
& \tilde{y}_{2}(t)=a_{1} \tilde{x}_{2}(t)+a_{2} \tilde{x}_{1}^{2}(t) \\
& \tilde{y}_{3}(t)=a_{1} \tilde{x}_{3}(t)+2 a_{2} \tilde{x}_{1}(t) \tilde{x}_{2}(t)+a_{3} \tilde{x}_{1}^{3}(t)
\end{aligned}
$$

Jede Antwort $\tilde{y}_{n}(t)$ des nichtlinearen Systems setzt sich dabei aus einem linearen Anteil $\tilde{y}_{n, \mathrm{~L}}(t)$ und einem nichtlinearen Anteil $\tilde{y}_{n, \mathrm{NL}}(t)$ zusammen.

$\tilde{y}_{n}(t)=\tilde{y}_{n, \mathrm{~L}}(t)+\tilde{y}_{n, \mathrm{NL}}(t)$

Der lineare Anteil $\tilde{y}_{n, \mathrm{~L}}(t)$ der Ausgangsgröße $\tilde{y}_{n}(t)$ ist eine lineare Funktion der n-ten Eingangsgröße $\tilde{x}_{n}(t)$, währenddessen der nichtlineare Anteil $\tilde{y}_{n, \mathrm{NL}}(t)$ eine nichtlineare 

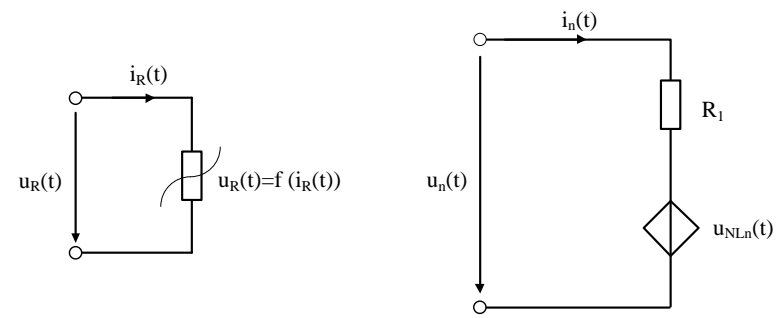

(a) Zeitinvariant

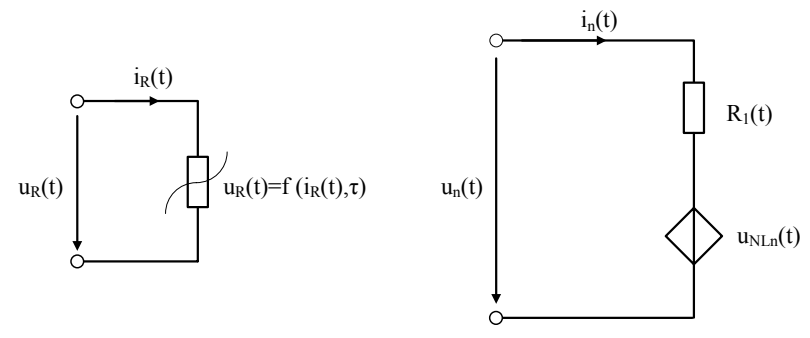

(b) Zeitvariant

Abb. 3. Nichtlinearer Widerstand (links) mit der dazugehörigen äquivalenten Volterra-Schaltung (rechts) für den (a) zeitinvarianten und (b) zeitvarianten Fall.

Funktion der niedrigeren Ordnungen der Eingangsgröße $\tilde{x}_{1}(t), \tilde{x}_{2}(t), \ldots, \tilde{x}_{n-1}(t)$ ist.

$$
\begin{aligned}
& \tilde{y}_{n, \mathrm{~L}}(t)=a_{1} \tilde{x}_{n}(t) \\
& \tilde{y}_{n, \mathrm{NL}}(t)=f\left(\tilde{x}_{1}(t), \tilde{x}_{2}(t), \ldots, \tilde{x}_{n-1}(t)\right)
\end{aligned}
$$

Ist das nichtlineare System noch zeitvariant, so werden die Taylor-Koeffizienten $a_{1}, a_{2}, \ldots, a_{N}$ zeitabhängig. Somit kann man die konstanten Taylor-Koeffizienten in Gln. (6) durch zeitabhängige Koeffizienten $a_{1}(t), a_{2}(t), \ldots, a_{N}(t)$ ersetzen.

Abbildung 3 zeigt für das Beispiel eines nichtlinearen Widerstandes das äquivalente Kleinsignal-Modell für die Ordnung $n$, wobei der Strom $i_{R}(t)$ als Eingangsgröße und die Spannung $u_{R}(t)$ als Ausgangsgröße angenommen wird. $R_{1}$ entspricht dem ersten Taylor-Koeffizienten der StromSpannungsbeziehung (linearer Anteil des Widerstandes). Die Herleitung der Modelle für andere Grundnichtlinearitäten ist äquivalent (Yuan und Opal, 2001).

\section{Verfahren zur direkten Berechnung von Spektralan- teilen bei schwach nichtlinearen Netzwerken}

Im Folgenden soll ein Verfahren vorgestellt werden, das es ermöglicht Spektralanteile der Zustandsgrößen in einem schwach nichtlinearen Netzwerk bei harmonischen Anregungen zu bestimmen. Im Gegensatz zu Verfahren, wo eine Eingangs/Ausgangs Beschreibung der Gesamtschaltung erforderlich ist (Terrovitis und Meyer, 2000) sind nur die Eingangs/Ausgangs Beschreibungen der einzelnen nichtlinearen Elemente im Netzwerk nötig. Dies erlaubt die Berechnung

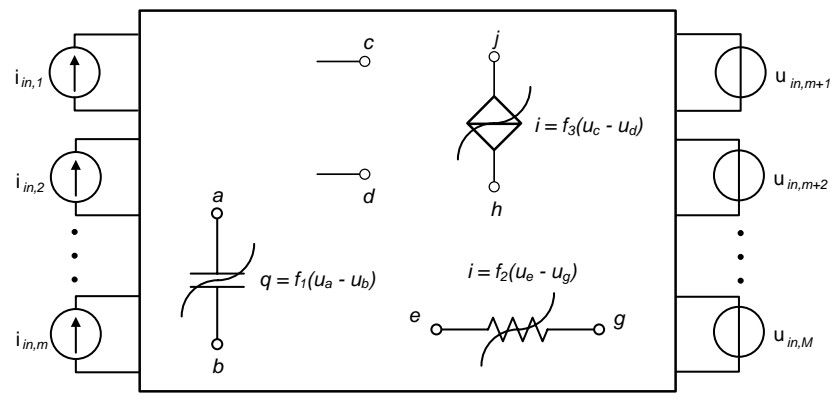

Abb. 4. Netzwerk mit nichtlinearen Elementen und mehreren Eingängen.

der Spektralanteile jedes Knotenpotentials und Zweigstromes innerhalb des Netzwerkes.

Im ersten Abschnitt dieses Kapitels wird das Verfahren allgemein für ein Netzwerk welches zeitinvariante schwach nichtlineare Elemente enthält beschrieben. Im zweiten Teil wird die Beschreibung auf Netzwerke mit periodisch zeitvarianten Elementen erweitert.

Wird ein Netzwerk, das schwach nichtlineare zeitinvariante Elemente enthält, mit mehreren harmonischen Quellen angeregt siehe Abb. 4, so besitzt jedes Knotenpotential und jeder Zweigstrom Spektralanteile bei Frequenzen bestehend aus Kombinationen der einzelnen Anregungsfrequenzen. Für das Potential $u_{k}(t)$ an dem $k$-ten Knoten und dem Strom $i_{z}(t)$ durch den $z$-ten Zweig gilt jeweils

$u_{k}(t)=\frac{1}{2} \sum_{x=1}^{X} U_{k, \omega_{x}} e^{j \omega_{x} t} \quad$ und $\quad i_{z}(t)=\frac{1}{2} \sum_{x=1}^{X} I_{z, \omega_{x}} e^{j \omega_{x} t}$,

wobei der Index $x$ die $x$-te Frequenzkombination kennzeichnet und die Zahl $X$ die Anzahl der möglichen Frequenzkombinationen angibt. Die Frequenz $\omega_{x}$ ist eine Linearkombination aus den Anregungsfrequenzen

$\omega_{x}=\sum_{m=1}^{M} a_{m} \omega_{m}$,

mit $a_{m} \in \mathbf{Z}$. $M$ entspricht der Anzahl der Anregungsfrequenzen. Die Koeffizienten $a_{m}$, für $m=1 . . M$, müssen die folgende Bedingung erfüllen (Bussgang et al., 1974)

$\sum_{m=1}^{M} a_{m}=N$,

wobei $N$ der Ordnung der Nichtlinearität des Netzwerkes entspricht. Da an jedem Knoten die kirchhoffsche Knotenregel gelten muss, kann an jedem Knoten eine Strombilanz bezüglich jeder Frequenz $\omega_{x}$ durchgeführt werden 


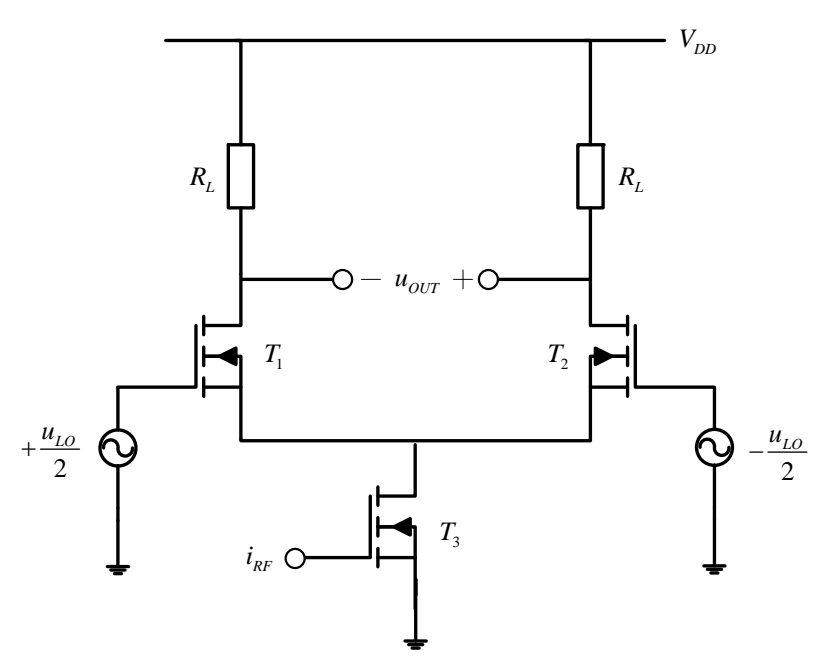

Abb. 5. Einfach balancierter Mischer.

$$
\sum_{z=1}^{Z} i_{z}\left(\omega_{x}\right)=0
$$

Nach der Methode der nichtlinearen Stromquellen (siehe Abschnitt 2) kann für jede Ordnung der Nichtlinearität $n$ jedes nichtlinearen Elementes im Netzwerk durch sein äquivalentes Kleinsignalmodell $n$-ter Ordnung ersetzt werden. Bei Aufstellung des Gleichungssystems für die entsprechende Ordnung werden die Zweigströme in Abhängigkeit der Knotenpotentiale und Netzwerkparameter ausgedrückt. Anschließend können nach Anwendung von Gln. (10) für jeden Zweigstrom im Netzwerk die Spektralanteile jedes Knotenpotentials ermittelt werden. Bei jeder Ordnung $n>1$ müssen dabei alle externe Anregungsquellen zu Null gesetzt werden.

Enthält das Netzwerk noch periodisch-zeitvariante nichtlineare Elemente, so müssen die Taylor-Koeffizienten der Eingangs-Ausgangs Beschreibung jedes zeitvarianten Elementes über die Fourier-Reihe im Frequenzbereich ausgedrückt werden. Für den $n$-ten Taylor Koeffizient $a_{n}(t)$ gilt

$a_{n}(t)=\sum_{p=-\infty}^{+\infty} A_{n, p} e^{j p \omega_{\mathrm{b}} t}$

wobei $\omega_{\mathrm{b}}$ der Basisfrequenz des zeitvarianten Elementes entspricht und der Koeffizient $A_{n, p}$ den Fourier-Koeffizienten $p$-ter Ordnung des Taylor-Koeffizienten n-ter Ordnung darstellt. Die Basisfrequenz der Zeitvarianz jedes Elementes kann als eine zusätzliche Anregungsfrequenz im Netzwerk betrachtet werden. Dementsprechend muss für jede Ordnung Gln. (11) erfüllt werden. Diese Darstellung erlaubt es, die Spektralanteile der Mischfrequenzen aufgrund der Nichtlinearität und der Zeitvarianz zu bestimmen.

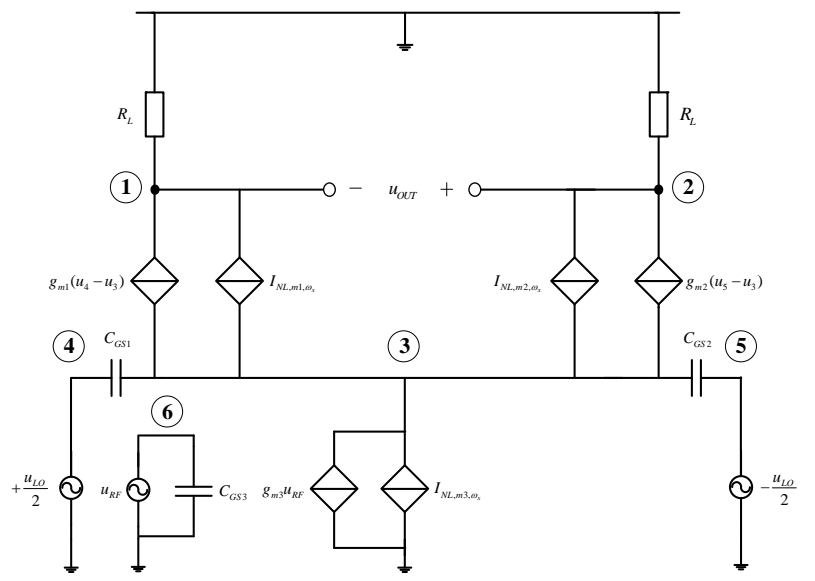

Abb. 6. Kleinsignal-Ersatzschaltbild n-ter Ordnung des einfach balancierten Mischers im nicht-schaltenden Betrieb.

\section{Nichtlinearitätsanalyse eines einfach balancierten MOS Mischers}

In diesem Kapitel soll die Anwendung des vorgestellten Verfahrens auf einem einfach balancierten MOS Mischer präsentiert werden. Hierbei werden zwei Betriebsfälle unterschieden: der nicht-schaltende Fall und der schaltende Fall. Für beide Betriebsfälle werden die Spektralanteile der Ausgangsspannung des Mischers bei jeder Ordnung der Nichtlinearität semi-symbolisch in Abhängigkeit der Entwurfs- und Prozessparameter ermittelt. Anschließend werden die Ergebnisse der Berechnungen diskutiert und gegenüber Simulationen mit SpectrRF verglichen.

\subsection{Die Mischerschaltung und das MOSFET-Modell}

Die Mischerschaltung ist in Abb. 5 gezeigt. Es handelt sich dabei um einen einfach balancierten MOS Mischer mit zwei verschiedenen Eingängen. Die nichtlinearen Bauelemente der Schaltung sind die MOSFETs T1 und T2 der LO-Stufe und der MOSFET T3 der Eingangsstufe. Um eine symbolische Analyse der Schaltung zu ermöglichen, muss ein analytisches Transistor-Modell verwendet werden. Für jeden Transistor wird das folgende Modell gewählt:

$I_{\mathrm{D}}=K_{n} \frac{U_{\mathrm{OD}^{2}}}{1+\left(\theta U_{\mathrm{OD}}\right)}$

mit $K_{n}=\frac{1}{2} \frac{W}{L} \mu_{0} C_{\mathrm{ox}}$, wobei $W$ und $L$ die Kanalbreite und Kanallänge sind. $C_{\mathrm{OX}}$ ist die Gateoxid-Kapazität pro Flächeneinheit und $\mu_{0}$ ist die Oberflächenbeweglichkeit. $\theta$ bezeichnet die Reduzierung der Ladungsträgerbeweglichkeit. $U_{\mathrm{OD}}$ ist die Overdrivespannung $\left(U_{\mathrm{GS}}-U_{\mathrm{TH}}\right)$. 


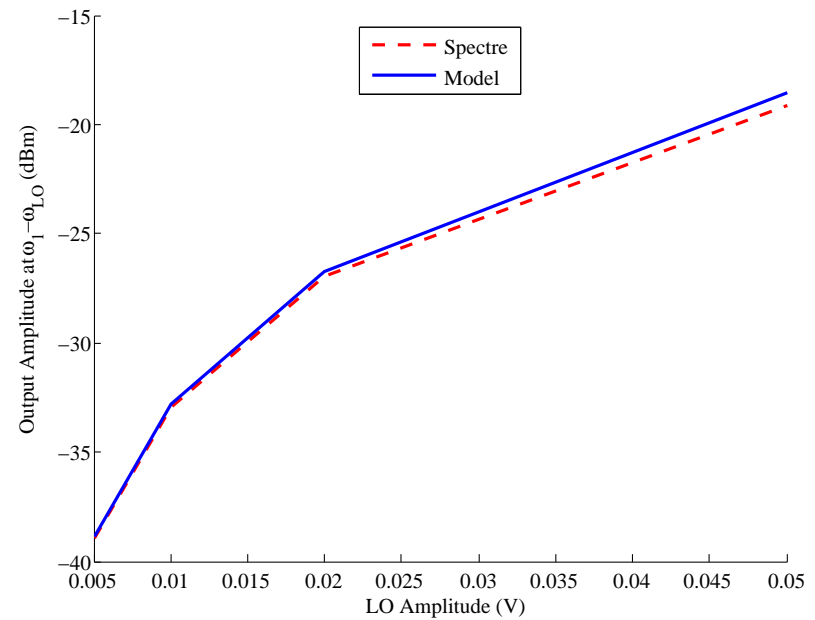

Abb. 7. Amplitude des Spektralanteils der Ausgangsspannung bei $\omega_{1}-\omega_{\text {LO }}$ gegen LO-Amplitude im nicht schaltenden-Betrieb.

\subsection{Ermittlung der Spektralanteile}

\subsubsection{Nicht-schaltender Betrieb}

Der erste Schritt bei der Anwendung des Verfahrens ist das Kleinsignalmodell $n$-ter Ordnung der Schaltung zu erstellen. Das Kleinsignal-Ersatzschaltbild $n$-ter Ordnung der Schaltung für den nicht-schaltenden Betriebsfall ist in Abb. 6 gezeigt. Über die modifizierte Knotenpunktanalyse (MNA) ergibt sich das folgende Gleichungssystem für die Schaltung

$\mathbf{Y} \cdot \boldsymbol{U}=\boldsymbol{I N} \triangleq$

$$
\begin{aligned}
& \left(\begin{array}{ccc}
G_{L} & 0 & g_{m 1} \\
0 & G_{L} & g_{m 2} \\
0 & 0 & -\left(g_{m 1}+g_{m 2}\right)+\left(C_{g s 1}+C_{g s 2}\right) \frac{d}{d t}
\end{array}\right) \cdot\left(\begin{array}{l}
u_{1} \\
u_{2} \\
u_{3}
\end{array}\right) \\
& =\left(\begin{array}{c}
\frac{g_{m 1} u_{L O}}{2}+I_{N L 1, n} \\
\frac{g_{L O}\left(g_{m 2}-g_{m 1}\right)}{2}+\frac{\left(C_{g s 1}-C_{g s 2}\right)}{2}+\frac{d u_{L O}}{d t}-g_{m 3} u_{R F}-I_{N L s, n}
\end{array}\right)
\end{aligned}
$$

wobei $u_{k}$ das Potential an den Knoten $k$ darstellt, $G_{\mathrm{L}}=\frac{1}{R L}$ und $I_{\mathrm{NL}, n}=I_{\mathrm{NL} 1, n}+I_{\mathrm{NL} 2, n}+I_{\mathrm{NL} 3, n}$.

Zur Bestimmung der Spektralanteile an jedem Knotenpotential wird zuerst die erste Ordnung behandelt. Da die nichtlinearen Stromquellen $I_{\mathrm{NL} 1,1}, I_{\mathrm{NL} 2,1}$ und $I_{\mathrm{NL} 3,1}$ gleich Null sind, stehen im Anregungsvektor IN nur noch die externen Anregungsquellen. Anschließend wird das Gleichungssystem für jede Anregungsfrequenz $\omega_{m}, m=1 . . M$, der externen Anregungsquellen gelöst (vgl. Gln. 12).

$\mathbf{Y}\left(\omega_{m}\right) \cdot \boldsymbol{U}\left(\omega_{m}\right)=\boldsymbol{I} \boldsymbol{N}\left(\omega_{m}\right)$

Nach Bestimmung der Spektralanteile der ersten Ordnung $\omega_{m}$ für $m=1 . . M$ an jedem Knoten, kann nun das Gleichungssystem für die zweite Ordnung $n=2$ gelöst werden. Zunächst werden die externen Anregungsfrequenzen zu Null

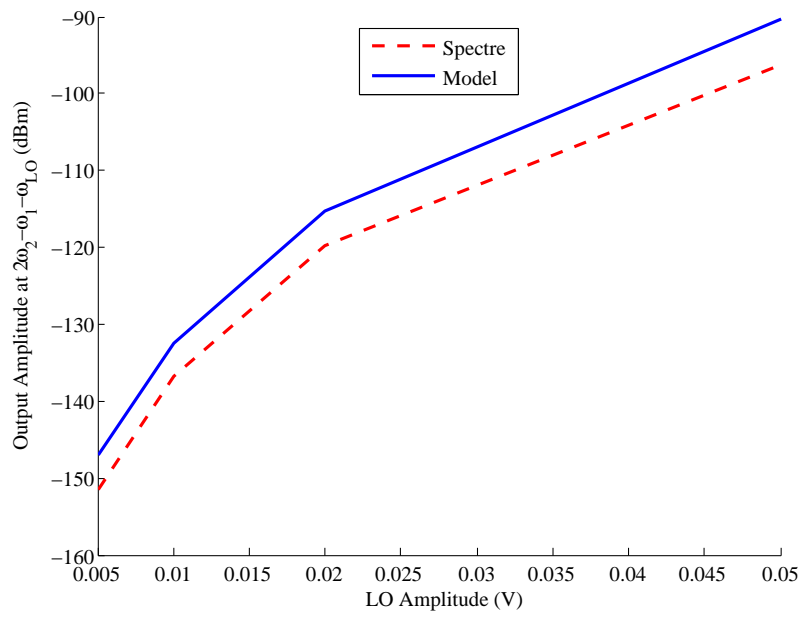

Abb. 8. Amplitude des Spektralanteils der Ausgangsspannung bei $2 \omega_{1}-\omega_{2}-\omega_{\text {LO }}$ gegen LO-Amplitude im nicht-schaltenden Betrieb.

gesetzt und die nichtlinearen Stromquellen 2-ter Ordnung $I_{\mathrm{NL} 1,2}, I_{\mathrm{NL} 2,2}$ und $I_{\mathrm{NL} 3,2}$ aus den Antworten erster Ordnung bestimmt (vgl. Gln. 7). Zur Bestimmung eines Kontenpotentials bei der Frequenz $\omega_{p}+\omega_{q}$ müssen die Knotenpotentiale bei den Frequenzen $\omega_{p}$ und $\omega_{q}$ in der Analyse erster Ordnung bestimmt werden. Zur Bestimmung der Spektralanteile der 3-ten und höheren Ordnungen wird die gleiche Vorgehensweise durchgeführt.

\subsubsection{Schaltender Betrieb}

Bei dem schaltenden Betrieb führt die große Amplitude des LO-Signals zum Schalten der Transistoren T1 und T2. Da in diesem Fall das System bzgl. dem LO-Eingang stark nichtlinear ist, ist eine Analyse des Systems mit Annahme einer schwachen Nichtlinearität unzulässig. Um dieses Problem zu lösen wird das LO-Signal als Teil des Systems betrachtet. Somit kann die Schaltung als ein zeitvariantes schwach nichtlineares System bzgl. des RF-Eingangs modelliert werden.

Die Anwendung des Verfahrens zur Bestimmung der Spektralanteile bei dem schaltenden Mischer hat die gleiche Vorgehensweise wie bei dem Fall des nicht-schaltenden Mischers. Es ergeben sich jedoch zwei Ausnahmen. Erstens wird das LO-Signal nicht mehr als eine externe Anregungsquelle behandelt. Zweitens ist eine Großsignalanalyse zur Bestimmung des zeitvarianten Arbeitspunktes jedes Elementes vorausgesetzt. Eine Taylorreihenentwicklung der Eingangs-Ausgangs Beschreibung des nichtlinearen Elementes um den zeitvarianten Arbeitspunkt ergibt zeitabhängige Taylor-Koeffizienten, die gemäß Gln. (13) in einer FourierReihe entwickelt werden können. Da laut dieser Modellierung die Transistoren T1 und T2 zeitvariante Arbeitspunkte 


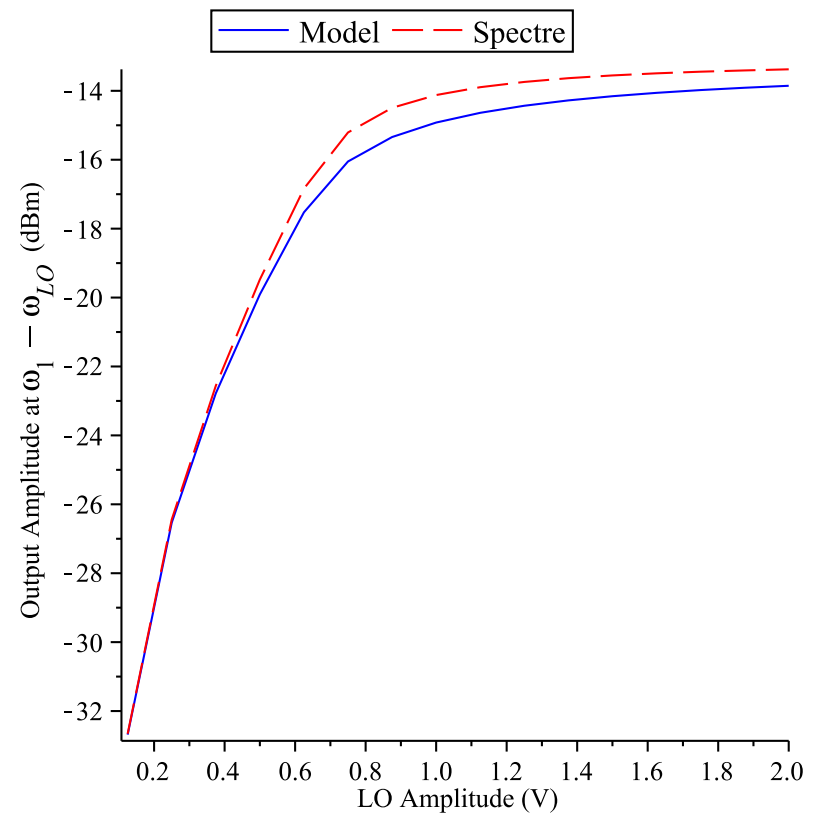

Abb. 9. Amplitude des Spektralanteils der Ausgangsspannung bei $\omega_{1}-\omega_{\text {LO }}$ gegen der LO-Amplitude im schaltenden Betrieb.

besitzen gilt für beide Transistoren

$g_{m}(t)=\sum_{k=-\infty}^{+\infty} G_{m, k} e^{j k \omega_{\mathrm{LO} t}}$

Bei Vernachlässigung der Kanallängenmodulation bleibt der Arbeitspunkt von Transistor T3 konstant. Da die nichtlinearen Stromquellen n-ter jedes nichtlinearen Elementes von den Taylor-Koeffizienten niedrigerer Ordnungen abhängen, sind auch die nichtlinearen Stromquellen der Transistoren T1 und $\mathrm{T} 2$ zeitabhängig.

\subsubsection{Ergebnisse der Berechnungen}

Das Verfahren wurde für den einfach balancierten Mischer in MAPLE implementiert. Berechnungen der Spektralanteile an jedem Knotenpotential wurden durchgeführt und semi-symbolische Ausdrücke für diese in Abhängigkeit der Entwurfs- und Prozessparameter ermittelt. Als Beispiel ist die Amplitude des Spektralanteils an Knoten 3 bei der Frequenz $\omega_{1}-\omega_{\mathrm{LO}}$ für den nicht-schaltenden Betrieb:

$u_{\text {out }}\left(\omega_{1}-\omega_{L O}\right)=\frac{g_{m 3} A_{\text {in }} A_{L O}\left(K_{2 g m 1} g_{m 2}-K_{2 g m 2} g_{m 1}\right)}{\left(g_{m 1}+g_{m 2}\right)^{3}}$

$A_{\text {in }}$ ist die Eingangsamplitude, $A_{L O}$ ist die Amplitude des LO-Signals und $K_{2 g m}$ ist der Taylor-Koeffizient zweiter Ordnung des Transistors.

Abbildungen 7 und 8 zeigen die Verläufe der Spektralanteile der Ausgangsspannung $u_{\text {out }}=u_{2}-u_{1}$ an den Frequen-

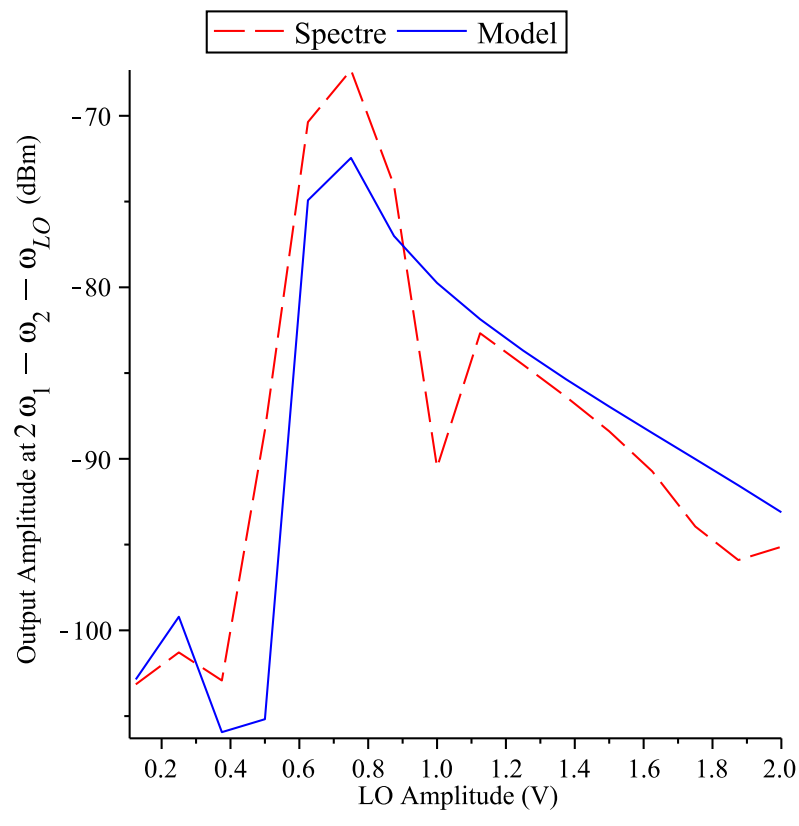

Abb. 10. Amplitude des Spektralanteils der Ausgangsspannung bei $2 \omega_{1}-\omega_{2}-\omega_{\text {LO }}$ gegen der LO-Amplitude im schaltenden Betrieb.

zen $\omega_{1}-\omega_{\mathrm{LO}}$ und $2 \omega_{1}-\omega_{2}-\omega_{\mathrm{LO}}$ bei einer Zweitonanregung $\left(\omega_{1}, \omega_{2}\right)$ am RF-Eingang im nicht-schaltenden Fall. Die Ergebnisse der Berechnungen zeigen gute Übereinstimmungen gegenüber der Simulationen mit SpectreRF. In Abb. 7 ist zu sehen, dass die Amplitude des abwärtsgemischten Signal mit der LO-Amplitude steigt. Dies ist aufgrund der Multiplikation des RF-Signals mit der Amplitude des LO-Signals. Die Amplitude des Spektralanteils aus der Intermodulation 3. Ordnung nimmt auch mit der LO-Amplitude zu (Abb. 8), da die Nichtlinearität im System mit größerer LO-Amplitude stärker ausgesteuert wird.

Die Ergebnisse der Berechnungen für den schaltenden Fall sind in den Abb. 9 und 10 gezeigt. Die Ergebnisse zeigen gute Übereinstimmungen gegenüber der Simulationen mit SpectreRF. Wie im nicht-schaltenden Fall steigt die Amplitude des abwärtsgemischten Signals mit der LO-Amplitude (vgl. Abb. 9). Da mit höheren LO-Amplituden die Zeitabschnitte, in den die beiden Transistoren T1 und T2 zusammen aktiv sind, abnehmen, nimmt der Einfluss der Nichtlinearität beider Transistoren auf das Ausgangsverhalten ab. Dies ist auch in Abb. 10 zu sehen, wo die Amplitude des Spektralanteils aus der Intermodulation 3. Ordnung mit der LO-Amplitude abnimmt.

\section{Zusammenfassung}

In dieser Arbeit wurde ein Verfahren zur Bestimmung der Spektralanteile der Zustandsgrößen in einem Mischer basierend auf der Methode der nichtlinearen Stromquellen vorgestellt. Im Gegensatz zu Verfahren, wo eine 
Eingangs-Ausgangs Beschreibung des Gesamtsystems erforderlich ist, handelt es sich hier um ein elementbasiertes Verfahren. Dies erlaubt die Berechnung der Spektralanteile jedes Knotenpotentials sowie Zweigstromes in der Schaltung. Ein wesentlicher Vorteil des Verfahrens ist die Möglichkeit semi-symbolische Ausdrücke für die Spektralanteile herzuleiten. Das Verfahren wurde auf einfach balancierte Mischer, sowohl im schaltenden als auch nicht-schaltenden Betrieb angewandt. Die Implementierung des Verfahrens in dem Computeralgebraprogramm MAPLE erlaubte die Bestimmung semi-symbolischer Ausdrücke für die Spektralanteile in Abhängigkeit der Entwurfs- und Prozessparameter. Die erzielten numerischen Ergebnisse zeigten gute Übereinstimmungen mit den Simulationen aus SpectrRF.

\section{Literatur}

Bedrosian, E. und Rice, O.: The output properties of Volterra systems (nonlinear systems with memory) driven by harmonic and Gaussian inputs, Proc. IEEE, 59(12), 1688-1707, 1971.

Bussgang, J. J. Ehrman, L., und Graham, J. W.: Analysis of nonlinear systems with multiple inputs, Proceedings of the IEEE, 62(8), 1088-1119, 1974.
Flake, R. H.: Volterra series representation of time-varying nonlinear systems in Proc. Second Int. Congress of IFAC on Automatic Control, paper no. 408/1, 1963.

Maas, S. A.: Two-Tone Intermodulation in Diode Mixers, Microwave Theory and Techniques, IEEE Transactions on, 35(3), 307314, 1987.

Oberg, H. J.: Berechnung nichtlinearer Schaltungen für die Nachrichtenübertragung, Teubner Studienskripten, Stuttgart 1973.

Schetzen, M.: The Volterra and Wiener Theories of Nonlinear Systems, Wiley-Interscience, 1980.

Schetzen, M.: Multilinear Theory of Nonlinear Networks, The Franklin Institute 0016-0032/85, 1985.

Swerdlow, R. B.: Analysis of Intermodulation Noise in Frequency Converters by Volterra Series, Microwave Theory and Techniques, IEEE Trans, 26(4), 305-313, 1978.

Terrovitis, M. T. und Meyer, R. G.: Intermodulation distortion in current-commutating CMOS mixers, Solid-State Circuits, IEEE Journal of, 35(10), 1461-1473, 2000.

Wambcaq, P. und Sansen, W.: Distortion Analysis of Analog Integrated Circuits, Kluwer Academic Publishers, 1998.

Yuan, F. und Opal, A.: Distortion analysis of periodically switched nonlinear circuits using time-varying Volterra series, Circuits and Systems I: Fundamental Theory and Applications, IEEE Transactions on, 48(6), 726-738, 2001. 